\title{
Hernia de Amyand tipo 2: apendicectomía y hernioplastia con malla de baja densidad
}

\author{
Amyand's hernia type 2: appendectomy and hernioplasty with \\ low density mesh
}

\author{
Jorge Luis Vélez-Bernal' ${ }^{1}$ D, Juan Sebastián Martínez-Buitrago² (D, \\ Leidy Carolina García-Mendieta ${ }^{3-4} \mathbb{D}$, Cristina Estefanía González-Díaz $\mathbb{D}^{4}$
}

\begin{abstract}
Médico, especialista en Cirugía general, Hospital Central de la Policía Nacional, Bogotá, D.C. Colombia. Médico, especialista en Epidemiología, Hospital Central de la Policía Nacional, Bogotá, D.C. Colombia.

3 Médico, magister en morfología humana, Hospital Central de la Policía Nacional; profesor instructor de anatomía, Universidad de Ciencias Aplicadas y Ambientales, UDCA, Bogotá, D.C. Colombia.

4 Médico, Hospital Central de la Policía Nacional, Bogotá, D.C. Colombia.
\end{abstract}

\section{Resumen}

La incidencia de las hernias inguinales en Colombia es de aproximadamente 15 por cada 1000 habitantes, y el riesgo de presentar una hernia inguinal durante la vida es del $27 \%$ en hombres y del $3 \%$ en mujeres. Existen presentaciones poco frecuentes como la hernia de Amyand que consiste en la protrusión del apéndice cecal, inflamada o no, dentro del contenido del saco herniario. A nivel global, tiene una incidencia del 0,13\% al $1 \%$ y su tratamiento se realiza basándose en la clasificación de Losanoff y Basson. Sin embargo, una de las controversias aún existentes respecto al manejo quirúrgico, es la indicación del uso o no de mallas al momento de realizar la hernioplastia y el resultado final del mismo, así que este es el punto donde se centra la revisión de nuestro artículo. Se presenta el caso de un paciente de 79 años con una hernia de Amyand tipo 2, a quien se le practicó hernioplastia con malla de baja densidad absorbible, con adecuada evolución posquirúrgica.

Palabras clave: hernia inguinal; hernia de Amyand; apendicectomía; herniorrafia; prótesis e implantes.

\begin{abstract}
The incidence of inguinal hernias in Colombia is approximately 15 per 1000 inhabitants, and the risk of presenting an inguinal hernia during life is $27 \%$ in men and $3 \%$ in women. There are rare presentations such as Amyand's hernia, which consists of the protrusion of the cecal appendix, inflamed or not, within the content of the hernial sac. At a global level, it has an incidence of $0.13 \%$ to $1 \%$ and its treatment is based on the Losanoff and Basson

Fecha de recibido: 02/09/2019 - Fecha de aceptación: 27/02/2020

Correspondencia: Cristina Estefanía González-Díaz, Calle 74 No 89 - 12, Bogotá, D.C., Colombia. Teléfono: 3168734654.

Correo electrónico: cristina01_09@hotmail.com

Citar como: Vélez-Bernal JL, Martínez-Buitrago JS, García-Mendieta LC, González-Díaz CE. Hernia de Amyand tipo 2: apendicectomía y hernioplastia con malla de baja densidad. Rev Colomb Cir. 2021;36:150-4. https://doi.org/10.30944/20117582.463

Este es un artículo de acceso abierto bajo una Licencia Creative Commons - BY-NC-ND https://creativecommons.org/licenses/by-ncnd/4.0/deed.es
\end{abstract}


classification. However, one of the still existing controversies regarding surgical management is the indication of the use or not of meshes at the time of performing the hernioplasty, and the final result of it, this is the point where the review of our article focuses. We present the case of a 79-year-old patient with a type 2 Amyand hernia who underwent hernioplasty with a low-density absorbable mesh, with adequate postoperative evolution.

Keywords: hernia, inguinal; Amyand's hernia; appendectomy; herniorrhaphy; prostheses and implants.

\section{Introducción}

Una de las patologías más frecuentes de la pared abdominal es la hernia inguinal. En países con alta densidad poblacional como Estados Unidos, se realizan más de medio millón de intervenciones anuales, por lo cual, es uno de los procedimientos más comúnmente realizados por parte de los cirujanos generales ${ }^{1}$.

Se conoce como hernia de Amyand, a una hernia inguinal a través de la cual protruye el apéndice cecal dentro del contenido del saco herniario, estando o no inflamada ${ }^{2-4}$. Presenta una incidencia del 0,4-1\% respecto al total de hernias y en caso de apendicitis del 0,1\%. Tiene una mayor frecuencia en hombres y distribución bimodal en neonatos y en mayores de 70 años ${ }^{4-6}$.

El tratamiento de las hernias de Amyand, se basa actualmente en la clasificación propuesta por Losanoff y Basson, donde se plantea la apendicectomía en los casos que presentan inflamación, perforación o gangrena ${ }^{6,7}$. Una de las controversias aún existentes al respecto, es el manejo quirúrgico con o sin el uso de mallas, y el resultado final del mismo, aspecto en el que se centra la revisión de nuestro artículo.

\section{Presentación de caso}

Paciente masculino de 79 años, quién ingresó al servicio de urgencias por dolor inguinal derecho, irradiado al miembro inferior ipsilateral, y que se exacerbó en las últimas 24 horas, sin presentar otros síntomas. Al examen físico, se encontró edema de pared abdominal, eritema en región inguinal derecha asociado a dolor, y masa no reductible, por lo cual se hizo el diagnóstico de hernia encarcelada. Se practicó una ultrasonografía de abdomen, en la que se observó, disminución del peristaltismo, asas intestinales dilatadas con abundante gas y líquido intraluminal de predominio en fosa iliaca derecha. La ultrasonografía de tejidos blandos informó un defecto de continuidad de la fascia en la región inguinal derecha de 2,2 mm con protrusión de un saco herniario compuesto por grasa y asas intestinales no reductibles.

El paciente fue llevado a cirugía, practicando una incisión para-inguinal, con reducción del contenido herniario, encontrando una hernia inguinal L1 primaria, según la clasificación de la Sociedad Europea de la Hernia (EHS, por sus siglas en inglés), encarcelada, con apéndice cecal contenida en el saco herniario y en fase gangrenosa (figuras 1, 2 y 3), por lo que se clasificó como una hernia de Amyand tipo 2 según la clasificación de Losanoff y Bansson (tabla 1).

Se revisó el resto de la cavidad abdominal sin encontrar otros hallazgos relevantes. Se realizó la corrección quirúrgica, por técnica extraperitoneal inguinal, empleando una malla de polipropileno de baja densidad (ultrapro ${ }^{\circledR}$ ), de 10 X 15 centímetros. El paciente recibió terapia antibiótica por cinco días, presentó una evolución posquirúrgica adecuada y sin complicaciones, con controles posoperatorios normales.

\section{Discusión}

La hernia de Amyand fue descrita originalmente en 1735 por Claudius Amyand quien durante el tratamiento de una hernia inguinal encarcelada en un niño de 11 años, encontró el apéndice cecal perforada dentro del saco herniario, por lo que debió realizar una apendicectomía ${ }^{7,8}$. En 1731 Jacques Croissente de Garengeot había descrito la presencia del apéndice cecal, contenida en el saco de una hernia femoral ${ }^{8}$. La incidencia es del $0,13-1 \%$ a nivel global y como complicación asociada, se pueden encontrar la perforación del apéndice dentro del saco herniario ${ }^{8}$. 

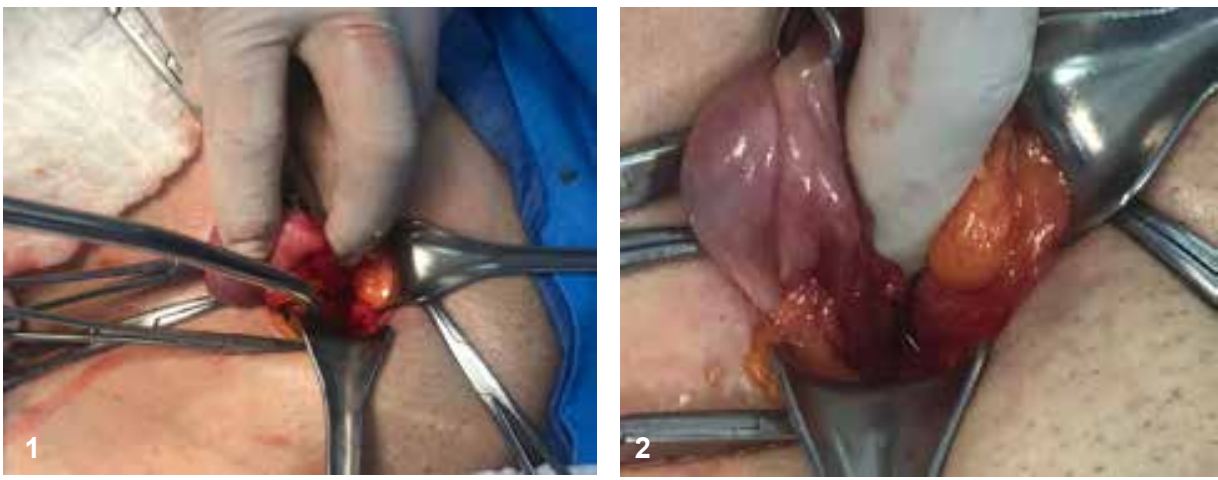

Figuras 1 y 2. Región inguinal derecha. Se observa el apéndice cecal contenida en el saco herniario (1) y el lipoma herniario (2).

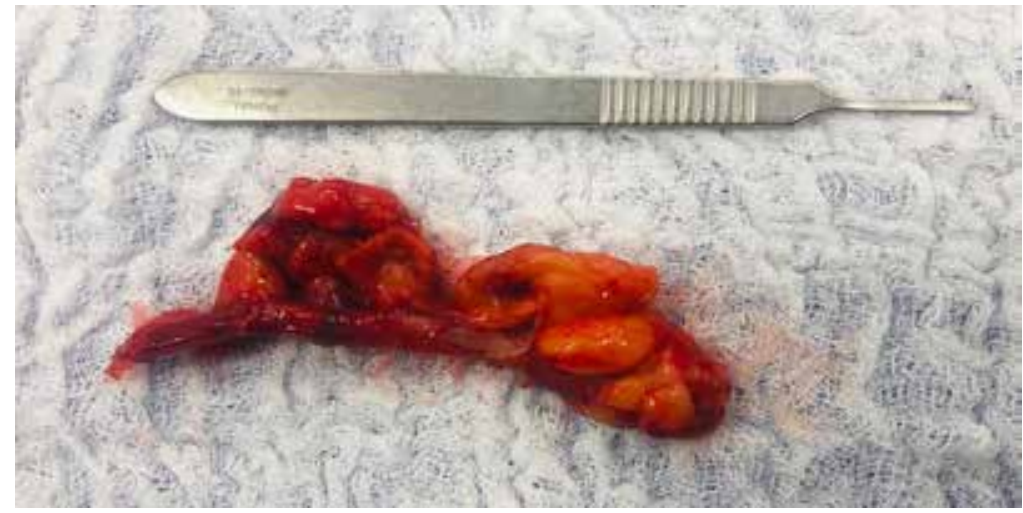

Figura 3. Apéndice cecal en fase gangrenosa.

Tabla 1. Clasificación de Losanoff y Basson para la hernia de Amyand

\begin{tabular}{lllll}
\hline & \multicolumn{1}{c}{ Tipo 1 } & \multicolumn{1}{c}{ Tipo 2 } & \multicolumn{1}{c}{ Tipo 3 } & \multicolumn{1}{c}{ Tipo 4 } \\
\hline Descripción & $\begin{array}{l}\text { Apéndice normal en una } \\
\text { hernia inguinal }\end{array}$ & $\begin{array}{l}\text { Apendicitis aguda en } \\
\text { una hernia inguinal } \\
\text { sin sepsis abdominal }\end{array}$ & $\begin{array}{l}\text { Apendicitis aguda en } \\
\text { una hernia inguinal y } \\
\text { sepsis peritoneal o de } \\
\text { pared abdominal }\end{array}$ & $\begin{array}{l}\text { Apendicitis aguda en } \\
\text { una hernia inguinal con } \\
\text { enfermedad abdominal } \\
\text { concomitante }\end{array}$ \\
\hline Tratamiento & $\begin{array}{l}\text { Reducción de la hernia y y } \\
\text { colocación de malla }\end{array}$ & $\begin{array}{l}\text { Apendicectomía y re- } \\
\text { paración primaria de } \\
\text { la hernia sin malla }\end{array}$ & $\begin{array}{l}\text { Laparotomía, apendi- } \\
\text { cectomía y reparación } \\
\text { de la hernia sin prótesis } \\
\text { o malla }\end{array}$ & $\begin{array}{l}\text { lgual que la } 3 \text { y trata- } \\
\text { miento de la enfermedad } \\
\text { concomitante }\end{array}$
\end{tabular}

La fisiopatología de la hernia de Amyand no es del todo clara, la teoría más aceptada menciona una variación anatómica poco frecuente, en donde el apéndice es largo, retro cecal y extra peritoneal, con un descenso marcado del ciego a la fosa iliaca derecha, con el apéndice localizado cerca al anillo profundo, lo que favorece su deslizamiento junto con el saco herniario, hacia el canal inguinal. En lugar de la obstrucción intraluminal del apéndice, la causa de la inflamación del apéndice en el saco 
herniario, usualmente está dada por la obstrucción extra luminal, debido a la contracción de los músculos abdominales, con el consecuente aumento de la presión abdominal ${ }^{9,10}$.

Uno de los diagnósticos diferenciales que se debe tener en cuenta, es la hernia de Littré, descrita por Alexis Littré en el año 1700, y que se caracteriza por involucrar la presencia de un divertículo de Meckel (divertículo verdadero, que se forma por la falta de obliteración del conducto ónfalo mesentérico, en la semana 15 de gestación) en el saco herniario. Esta hernia se localiza en el $50 \%$ de los casos en la región inguinal, en un $30 \%$ en el ombligo y en un $20 \%$ compromete la localización femoral ${ }^{11}$. El divertículo de Meckel se encuentra en el borde antimesentérico del íleon a $10-15 \mathrm{~cm}$ de la válvula ileocecal, con un diámetro de 4 - 6 centímetros, y tiene una incidencia de aparición del $2-3 \%$. Yamaguchi reportó en 1978 que, el 4,7 \% hacían parte del contenido del saco herniario que podría estar en los lugares anteriormente señalados. Se considera una patología poco frecuente, de la cual solo se han descrito cerca de 50 casos $^{12}$.

En la gran mayoría de los casos, el diagnóstico de la hernia de Amyand, se realiza de forma intraoperatoria, aunque se han documentado casos donde el diagnóstico se realiza de forma preoperatoria, por medio de la tomografía computarizada (TC), ${ }^{12,13,14}$.

Para orientar el manejo y según los hallazgos, se han diseñado tablas de clasificación, como por ejemplo la de Losanoff y Basson ${ }^{13,14}$. El manejo quirúrgico de la hernia de Amyand está claro para los tipos 1, 3 y 4 (tabla 1), siendo el uso de malla el manejo indicado para la corrección quirúrgica de la hernia inguinal tipo 1 , y estando contraindicado su uso en las hernias de tipo 3 y $4^{14}$. Actualmente, existe una controversia mundial en cuanto al manejo de las hernias tipo 2 , pero cada vez son más los reportes y series de casos, donde se describe el uso de mallas para el manejo de las hernias tipo 2 de manera exitosa, sin aumentar el riesgo de infección del sitio operatorio o la aparición de fístulas por el uso de la misma ${ }^{12,13,14}$. En nuestro medio, ya se han descrito casos como los de Castro ${ }^{12} \mathrm{y}$ Villarreal ${ }^{13}$ con resultados exitosos en el manejo final del paciente.
En este aspecto, es claro y relevante mencionar la importancia de la experiencia del cirujano, la elección correcta del tipo de malla (idealmente de baja densidad y microporosa), y el compromiso inflamatorio de los tejidos blandos y de las estructuras dentro del canal inguinal.

Finalmente, consideramos que, para el resultado exitoso, es fundamental una buena técnica quirúrgica, la estandarización del cuidado posquirúrgico y el seguimiento del paciente. Es muy probable que a futuro, como en otras áreas, el uso de la malla esté completamente indicado en el tratamiento de la hernia de Amyand tipo $2^{13,14,15}$.

\section{Conclusión}

La hernia de Amyand es una rara entidad, que combina dos patologías frecuentes a las cuales se debe enfrentar el cirujano en su práctica diaria. Se presentó el caso clínico de un paciente con hernia inguinal derecha encarcelada, con apendicitis en fase gangrenosa en el saco herniario, compatible con una hernia de Amyand tipo 2. Se realizó apendicectomía con reducción del saco herniario, y hernioplastia vía preperitoneal con técnica de Nyhus y colocación de una malla ultrapro ${ }^{\circledR}$ de baja densidad (monocryl ${ }^{\circledR}+$ prolene $^{\circledR}$ ). Esto, tiene la ventaja de evitar el contacto directo de la prótesis con el canal inguinal, disminuyendo el riesgo de la infección posoperatoria. Algunos pocos artículos mencionan que la mejor técnica quirúrgica es la de Bassini ${ }^{14,15}$.

En cuanto al reparo, con o sin malla, en procesos inflamatorios sin sepsis o sin foco infeccioso local, aún no se ha llegado a un consenso ${ }^{14,15}$, sin embargo, se considera que a pesar del riesgo aumentado de infección y rechazo de la malla, puede haber una mayor incidencia de recidiva sin el empleo de una prótesis ${ }^{15}$.

Se considera que el uso de malla, mediante un abordaje preperitoneal abierto o laparoscópico, evita el foco inflamatorio inguinal y disminuye la probabilidad de recidiva herniaria, técnica ya descrita en nuestro medio por el grupo de Villarreal $^{13}$. En las hernias tipo 3, debido al alto riesgo de infección de la prótesis, está contraindicado su uso ${ }^{15}$. 
Consideramos que, a pesar de ser una patología poco frecuente, es muy importante tenerla en cuenta. Se requieren más estudios que validen la utilidad del diagnóstico preoperatorio, así como el uso de mallas para la corrección de defecto herniario y la elección de la densidad de las mismas.

\section{Cumplimientos de normas éticas}

Consentimiento informado: Para la publicación de este caso, se obtuvo el consentimiento informado por parte del paciente.

Conflictos de interés. Los autores declaran no tener conflictos de interés en la elaboración del presente artículo.

Financiación: Autofinanciado por los autores.

Contribución de los autores: Concepción y diseño del estudio, adquisición de datos, análisis e interpretación de datos y redacción del manuscrito: Juan Sebastián Martínez-Buitrago, Leidy Carolina García-Mendieta, Cristina Estefanía González Díaz.

Concepción y diseño, revisión crítica y aprobación final: Jorge Luis Velez Bernal.

\section{Referencias}

1. Malangoni MA, Rosen MJ. Hernias. In: Townsend CM, Beauchamp RD, Evers BM, Mattox KL. Sabiston. Tratado de cirugía: Fundamentos biológicos de la práctica quirúrgica moderna. 19a edición. Barcelona, España: Elsevier; 2013. p. 1114-40.

2. Arce Aranda C, Rojas R, López L, Correa M, Sánchez R, Soskin Reidman A. Apéndice cecal en el saco herniario: hernia de Garengeot vs hernia de Amyand, presentación de casos clínicos. An Fac Cienc Méd Asunción. 2009;XLII:51-54.

3. Franko J, Raftopoulos I, Sulkowski R. A rare variation of Amyand's hernia. Am J Gastroenterology. 2002;97:2684-5.

https://doi.org/10.1111/j.1572-0241.2002.06060.x

4. Barut I, Tarhan ÖR. A rare variation of amyand's hernia: gangreneous appendicitis in an incarcerated inguinal hernia sac. Eur J Gen Med. 2008;5:112-4. https://doi.org/10.29333/ejgm/82588
5. Michalinos A, Moris D, Vernadakis S. Amyand's hernia: a review. Am J Surg. 2014;207:989-95. https://doi.org/10.1016/j.amjsurg.2013.07.043

6. Shaban Y, Elkbuli A, Mackenny M, Boneva D. Amyand's hernia: A case report and review of literature. Inter J Surg Case Rep. 2018;47:92-6. https://doi.org/10.1016/j.ijscr.2018.04.034

7. Morales Cárdenas A, Ploneda Valencia CF, Sainz Escárrega VH, Hernández Campos AC, Navarro Muñiz E, López Lizarraga CR, et al. Amyand hernia: Case report and review of the literature. Ann Med Surg. 2015;4:113e-5 https://doi.org/10.1016/j.amsu.2015.03.007

8. Burcu E, Ali C, Ishan A. Amyand's hernia: 11 years of experience. J Pediatric Surg. 2016;51:1327-9. https://doi.org/10.1016/j.jpedsurg.2015.11.010

9. Perroti S, Bosco D, Miano D, Amico A, Latino R, Di cataldo A. Incarcerated Amyand's hernia: A case report. Inter J Surg Case Rep. 2018;51:265-7. https://doi.org/10.1016/j.ijscr.2018.08.064

10. Scheidbach H, Blume B, Meyer F. Laparoscopic Herniotomia and Closure in Case of Appendicitis within the Hernia Sac (Amyand's hernia): Is Use of Mesh Contraindicated?. Zentralb Chir. 2017;142:312-31.

11. Bains HK, Agostinho N, Hamilton AE, Byrne Ch. What is in the sac? Littré hernia. ANZ J Surg. 2020;90:896-8. https://doi.org/10.1111/ans.15353

12. Castro D, Borráez AM, Farías AP, Muñoz LM. Variante de una hernia de Amyand. Rev Colomb Cir. 2016;30: 276-82.

13. Villarreal R, Luna- Jaspe C, Cabrera LF, Vinck E. Hernia de Amyand encarcelada, revisión de la literatura y reporte de un caso en una institución de tercer nivel en Bogotá. Rev Colomb Cir. 2016;31:283-8.

14. Servide-Staffolani MJ, Perfecto-Valero A, Cervera Aldama J, Anduaga Peña MF, García González JM, Colina Alonso A, et al. Controversias en el tratamiento de la hernia de Amyand. Rev Colomb Cir. 2018;33:107-10. https://10.30944/20117582.52

15. Agirre Etxabe L, Prieto Calvo M, García Etxebarría A, García González JM, Sarriugarte Lasarte A, Colina Alonso A. Hernia de Amyand (tipo 2 de Losanoff) diagnosticada preoperatoriamente y tratada mediante hernioplastia con malla biológica. Rev hispanoam hernia. 2014;2:169-72.

https://doi.org/10.1016/j.rehah.2014.04.003 